\title{
Observatorio Urbano Arquitectónico de Entornos Saludables OUAES
}

\section{Urban Architectural Observatory of Healthy Environments}

\author{
Irma Elisa Palacios Reyesa_Erika Benítez Malagón ${ }^{b}$-Daniel Alejandro Gómez Escotoc \\ José René Morgado Bustos ${ }^{\mathrm{d}}$-Héctor Saúl Castilla Moyado_eJerónimo Palomec Guzmán ${ }^{f}$ \\ a Responsable del Observatorio Urbano Arquitectónico de Entornos Saludables, \\ Facultad de Arquitectura, Universidad Veracruzana, Córdoba, México. \\ Correo: irpalacios@uv.mx
}

b Observatorio Urbano Arquitectónico de Entornos Saludables,

Facultad de Arquitectura, Universidad Veracruzana, Córdoba, México.

Correo: ebenitez@uv.mx

c Observatorio Urbano Arquitectónico de Entornos Saludables,

Facultad de Arquitectura, Universidad Veracruzana, Córdoba, México.

Correo: dangomez@uv.mx

d Observatorio Urbano Arquitectónico de Entornos Saludables,

Facultad de Arquitectura, Universidad Veracruzana, Córdoba, México.

Correo: rmorgado@uv.mx

e Observatorio Urbano Arquitectónico de Entornos Saludables, Facultad de Arquitectura, Universidad Veracruzana, Córdoba, México.

Correo: hcastilla@uv.mx

${ }^{f}$ Observatorio Urbano Arquitectónico de Entornos Saludables,

Facultad de Arquitectura, Universidad Veracruzana, Córdoba, México.

Correo:.jpalomec@uv.mx

Recibido: 16 de agosto de 2020

Aceptado: 15 de octubre de 2020

RESUMEN: El Observatorio Urbano Arquitectónico de Entornos Saludables OUAES es un espacio institucional, académico y confiable de insumos para el apoyo a la investigación. Con el objetivo de recopilar, monitorear, analizar y generar información estadística relacionada 


\section{Observatorios - Observatorio Urbano Arquitectónico de Entornos Saludables}

con la observación del fenómeno que producen las ciudades no saludables relacionada al proceso de resiliencia urbana y arquitectónica, compartiendo conocimiento especializado para entender las ciudades como sistemas sociales y urbanos que inciden directamente en la salud y bienestar de los habitantes. Teniendo un alcance territorial de observación de la Región 3 del Estado de Veracruz comprendida por la Zona Metropolitana Córdoba y la Zona Metropolitana Orizaba. El OUAES está adscrito a la Facultad de Arquitectura Córdoba de la Universidad Veracruzana. El Observatorio se conforma y funciona bajo la colaboración y asesoría de la Coordinación Universitaria de Observatorios (CUO) de la Universidad Veracruzana (UV); y se apega a la pertenencia y pertinencia del Plan estratégico 2017-2020 contribuyendo a las políticas institucionales. También, el OUAES tiene como objetivo estratégico de contribuir de manera confiable y valida con la información generada para contribuir al logro de las metas de los ODS 2030. Se trabajará con una metodología diseñada con estadística básica y derivada; con indicadores básicos, indicadores clave e indicadores extensivos. Salud integral, percepción espacial, ambientes saludables y componentes que se consideran para identificarlos que son: la esperanza de vida, la percepción de bienestar y la huella ecológica.

Palabras clave: Ambientes saludables; urbanismo; arquitectura; salud integral; percepción espacial.

ABSTRACT: The Urban Architectural Observatory of Healthy Environments OUAES is an institutional, academic an reliable space of inputs to support research. In order to collect, monitor, analyze and generate statistical information related to the observation of the phenomenon produced by unhealthhy cities related to the process of urban and architectural resilience, sharing specialized knowledge to understand cities as social and urban systems that directly affect the health and well-being of the inhabitants. Having a territorial scope of obsevation of Region 3 of teh State of Veracruz comprised by the Córdoba Metropolitan Area and Orizaba Metropolitan Area. The OUAES is attached to the Córdoba Architecture Faculty of the Veracruzana University. The Observatory is formed and functions under the collaboration and advice of the University Coordination of Observatories of the Veracruzana University; and adheres to the membership and relevance of the Strategic Plan 2017 - 20120, contributing to institutional policies. Likewise, the OUAES has the strategic objetive of contributing in a reliable and valid way whith the information generated to contribute to the achievement of the SDG 2030. It wil work with a methodology designed with basic and derived statistics; whith basic indicators, key indicators and extensive indicators. Comprehensive health, spatial perception, healthy environments and components that are considered to identify them, which are: life expectancy, the perception of well-being and the ecological footprint. 
Keywords:. Healthy Environments, Urbanism, Architecture, Comprehensive Health, Spatial Perception.

\section{Introducción}

$\mathrm{U}$ n Entorno Saludable es un espacio urbano o arquitectónico que mantiene un equilibrio del hábitat entre el bienestar físico y emocional; y su contexto espacial. Pero debido al crecimiento urbano y a los procesos de cambio en las ciudades se genera una desconexión entre los elementos y provocan una inestabilidad en diferentes factores; económico, social, ambiental y urbano arquitectónico. Este desequilibrio lo podemos observar directamente en los entornos a diferentes escalas urbanas y se pueden identificar como: espacios o entornos no saludables. Este tipo de entornos derivados del crecimiento de las urbes generan consecuencias e inciden en las conductas, los hábitos, el respeto, la armonía, la integración social, la seguridad, la identidad de los habitantes y problemas de salud.

En un contexto internacional y nacional hay un marco referencial y normativo que refieren diferentes documentos relacionados a los problemas de la salud asociados y contextualizados a la ciudad y a sus procesos urbanos de transformación; dentro de estos documentos y organismos identificados se pueden mencionar los siguientes: La carta de Atenas 1931, Informe Lalonde 1974, Carta de Ottawa 1986, los ODS de la Agenda 2030 de la ONU, SMAES (Asociación Mexicana de Arquitectos especialistas en la Salud), Happy Planet Index, INEGI, OMS. Todos estos referentes han sido de importancia y de sustento teórico para la conformación del Observatorio Urbano Arquitectónico de Entornos Saludables OUAES.

Desde la Carta de Atenas 1931 podemos observar referentes orientados hacia la salud asociados a las ciudades, a los espacios abiertos y cerrados tales como vialidades urbanas, espacios para la integración social, equipamiento urbano, espacios para la vivienda y espacios para la salud. La Carta de Atenas (1942) señala problemáticas y requerimientos referidos desde los años treinta y que aún siguen vigentes; refiere problemáticas en cuatro aspectos: del hábitat, del trabajo, de la recreación y circulación.

Dentro de estos aspectos refiere los siguientes factores de riesgo: desarrollo sin regulación de las ciudades sometidos a cambios continuos, concentración y alta densidad de población en las ciudades, caos y descontrol por hacinamiento, saturación de ciudades y abandono de las zonas rurales, consideraciones y problemáticas psicológicos, sociales y biológicos que se 


\section{Observatorios - Observatorio Urbano Arquitectónico de Entornos Saludables}

presentan por la influencia del medio, la situación geográfica, topográfica, situación social, política y económica; vivienda desordenada y descontrolada sin requerimientos de higiene, falta de áreas verdes, niveles de vida bajos, altas tasas de mortalidad, los espacios urbanos y arquitectónicos diseñados no siempre favorecen la salud, falta de servicios básicos.

Así también, podemos referir lo que nos menciona Marc Lalonde desde 1974 en un documento denominado Informe Lalonde en donde identifica cuatro factores que determinan el estado de la salud de los individuos: los factores, biológicos, factores ambientales y entorno, estilo de vida, organización de los sistemas de atención a la salud.

La Carta de Ottawa 1986 en donde define la promoción de la salud como: proporcionar a la población los medios necesarios para mejorar su salud y ejercer un mayor control sobre esta. Alcanzar un estado adecuado de bienestar físico, mental y social un individuo o grupo debe ser capaz de identificar y realizar aspiraciones, de satisfacer sus necesidades y de cambiar o adaptarse al ambiente.

Y menciona los requisitos previos para la salud, en donde cualquier mejora de la salud debe basarse en: paz, entendida como las condiciones de libertad y seguridad física de las personas; educación; vivienda digna, adecuada a las condiciones climáticas locales; alimentación sana y equilibrada; ingreso; ecosistema estable; justicia social y equidad. Y define cinco áreas de acción para la promoción de la salud: 1. Desarrollo de políticas públicas saludables; 2. creación de entornos propicios; 3. fortalecimiento de la acción comunitaria; 4. desarrollo de aptitudes personales; 5. Reorientación de los servicios de salud.

La Organización Mundial de la Salud (OMS),respecto a lo anterior, define que es evidente las desigualdades entre los diferentes núcleos de la población, principalmente los que viven en zonas de pobreza extrema ya que viven en entornos desfavorables, vivienda insalubre, en donde se identifican las condiciones de desigualdad y es en donde trascurren las actividades cotidianas de la población.

Dadas estas señalaciones, la OMS define los determinantes sociales de la salud como las circunstancias en las que las personas nacen, crecen, viven, trabajan y envejecen. Estas circunstancias están directamente ligadas a: aspectos económicos, políticos, sociales y espaciales en donde se involucran las decisiones en ámbitos globales, regionales, nacionales y locales. "El estilo de vida y las condiciones en donde transcurre la vida y trabajo de una persona, familia o grupo de personas determinan su grado de salud". 
Observatorios - Observatorio Urbano Arquitectónico de Entornos Saludables

Por eso, los entornos saludables para una población sana se ven condicionados por la ciudad, municipio o barrio en el que transcurre su vida y las condiciones en los que se encuentran los entornos. La OMS identifica mayor incidencia de enfermedades no transmisibles, catástrofes naturales, incidencia en variación de conductas sociales y emocionales de la población, hábitos, respeto, armonía, integración social, la seguridad y la identidad, daños al ambiente por la reducción de áreas verdes e incremento de contaminación atmosférica por la alta densidad de transporte y edificios.

Todos estos referentes mencionados son los que mueven al Observatorio Urbano Arquitectónico de Entornos Saludables a contribuir con información y monitoreo de este tipo de entornos y ciudades para poder aportar como arquitectos y urbanistas en las políticas y acciones que ayuden a tener y conservar ciudades que ofrezcan salud y bienestar a sus habitantes.

\section{Antecedentes y conformación del Observatorio Urbano Arquitectónico de Entornos Saludables OUAES}

La conformación del Observatorio Urbano Arquitectónico de Entornos Saludables (OUAES) surge como una iniciativa académica de investigación del cuerpo académico UV-CA-498 Arquitectura y Hábitat sustentable; orientado al desarrollo de una liga de investigación dirigida a la gestión urbano-arquitectónica, para un desarrollo del hábitat sustentable; en la cual observamos la importancia de la promoción de la salud en todos los entornos habitables que corresponden a los espacios urbanos y arquitectónicos.

Para la creación del OUAES se configuró un marco referencial organizado y estructurado de la siguiente manera: Marco teórico conceptual, marco normativo y un marco estadísticometodológico. En el primer marco se consideran los siguientes conceptos básicos: salud integral, percepción espacial, diseño de ambientes saludables, felicidad; y en lo que respecta al segundo que refiere el marco normativo, ayuda a alinear los objetivos que persigue el observatorio, considerando las siguientes organizaciones y documentos: OMS, SMAES, INEGI, ODS de la agenda 2030 de la ONU, Plan Estratégico UV 2017-2021 Pertenencia y Pertinencia, Sustainable Development Report 2019, SDG Index and Dashboard Report 2017. Y el tercero que corresponde al marco estadístico metodológico es el que soporta el diseño de los instrumentos de medición para generar los datos, interpretarlos y correlacionar los indicadores, constantes y variables que determinan el comportamiento del fenómeno de estudio. 


\section{Observatorios - Observatorio Urbano Arquitectónico de Entornos Saludables}

El inicio del OUAES surge del proyecto SIREI con número de registro 28848201872 denominado: "Observatorio académico de investigación denominado: Observatorio Urbano Arquitectónico de Entornos Saludables". El periodo del proyecto abarca del 21 de agosto 2018 al 21 de agosto 2020, tiene el objetivo de aproximarse al fenómeno de estudio: la identificación de los entornos no saludables; de generar y plantear una metodología e instrumentos de medición. Derivado de lo anterior se plantean dos escalas de abordar el tema: la escala Arquitectónica y la escala Urbana.

Producto del análisis de la escala Arquitectónica se desprende una publicación denominada: "Metodología aplicada: Una estrategia de diseño para generar ambientes universitarios saludables" publicado en la revista digital UVserva 6, en el 2018; así mismo, se genera la asesoría dirigida de cinco tesis de licenciatura aplicando la metodología en cinco Facultades de la Universidad Veracruzana ubicadas en la región tres del estado de Veracruz comprendida por la Zona Metropolita Orizaba y Zona Metropolitana Córdoba.

Respecto al estudio y análisis en escala Urbana, se presenta la ponencia denominada: "Aproximación metodológica para cálculo de indicadores en: Observatorio Urbano Arquitectónico de espacios saludables"; dentro del marco del 1er Congreso Internacional: Innovación docente multidisciplinaria, en donde se plantea la investigación en proceso de aplicación de la primera etapa a escala Urbana, etapa que se aplicó durante el periodo agosto 2019-enero 2020.

En el 2019 el Cuerpo académico logra obtener recursos a través de la convocatoria PROMEP 2019 de Fortalecimiento a Cuerpo Académico con el proyecto denominado: "Prototipos de experimentación como estrategia de aprendizaje en arquitectura y urbanismo aplicado en laboratorio de bioclimática experimental sustentable y en observatorio urbano arquitectónico de espacios saludables" aprobado el 16 de julio 2019, con vigencia a enero 2021.

Del proyecto anterior, surge como producto la ponencia y publicación en extenso del siguiente artículo: "Metodología de medición de habitabilidad urbana espacios saludables de Orizaba y Córdoba Veracruz"; presentado en el II Congreso Internacional: Espacios Urbanos, patrimoniales, turismo, habitabilidad y vida cotidiana en la Universidad Autónoma de Yucatán en colaboración con la Universidad de Guanajuato durante el mes de febrero del 2020 realizado en la ciudad de Mérida Yucatán, dicha ponencia y artículo que se presentó tiene el objetivo de mostrar los avances de la investigación PROMEP respecto al Observatorio OUAES; mostrando las seis encuestas diseñadas y aplicadas para la obtención y análisis de datos del fenómeno de estudio, que hasta el momento se tienen los resultados del 7.14\% de la región 
Observatorios - Observatorio Urbano Arquitectónico de Entornos Saludables

monitoreada correspondiente a la ZMC y ZMO; este porcentaje de monitoreo corresponde a 6 AGEBS de las dos ciudades principales de la Región 3 del estado de Veracruz.

Se continúa trabajando de manera colegiada entre el Cuerpo Académico UV-CA-498, y la Academia de Urbanismo de la licenciatura de Arquitectura en la Facultad de Córdoba; así como la integración y participación con las siguientes experiencias educativas: Prácticas profesionales, Servicio social, Experiencia recepcional, Diseño urbano: regeneración urbana, Preservación y conservación patrimonial. Con el único fin de contribuir en la toma de decisiones y de realización de proyectos urbanos que aporten a la generación de espacios y entornos de calidad. La aportación y objetivo principal del Observatorio Urbano Arquitectónico de Entornos Saludables respecto al fenómeno de estudio; será: monitoreo, generación y análisis de información construyendo una base de datos confiable para la generación y aplicación del conocimiento; contribuyendo a nuestra responsabilidad social con pertenencia y pertinencia, para la Universidad Veracruzana. Posicionándose como referente regional, nacional e internacional. Promoviendo la cultura del uso y aplicación de la estadística en la toma de decisiones y observación de problemáticas en casos de estudios y resolución de problemáticas urbanas y arquitectónicas.

\section{¿Porque crear un Observatorio Urbano Arquitectónico de Entornos Saludables OUAES?}

Con el paso del tiempo las ciudades han tenido procesos de cambio en diferentes aspectos como lo son morfología, legibilidad urbana, estructura urbana y en sus condiciones respecto a los diferentes factores que interactúan en ella, resultado de estos procesos de cambio se han generado ciudades y espacios no saludables; ante eso surge la necesidad de aportar y contribuir al programa mundial de ciudades saludables considerando el Plan Nacional de Desarrollo en donde está contemplado el Plan sectorial de salud y el Programa entornos y comunidades saludables que tienen la única finalidad de lograr entornos favorables a la salud.

Un entorno saludable es considerado como un espacio que apoya a la salud y favorece a una población (Latapí, 2015), La Sociedad Mexicana de Arquitectos Especializados en Salud (SMAES) como organismo especializado en observar la arquitectura y el espacio urbano desde el aspecto de salud; nos indica los aspectos del entorno que se deben considerar para influir positiva o negativamente en las personas que transitan, caminan o permanecen en los espacios abiertos o cerrados. La SMAES señala que: Los habitantes deben tener cubiertas todas sus necesidades básicas, su planeación debe ser continua y contemplada a largo plazo, 


\section{Observatorios - Observatorio Urbano Arquitectónico de Entornos Saludables}

la densidad demográfica debe ser equilibrada, la zonificación racional de funciones básicas como: habitación, trabajo, recreación y circulación, también indica que una ciudad saludable debe contar con áreas verdes estratégicamente diseñadas, con áreas de trabajo integradas y bien ubicadas con cordones o barreras verdes que protejan todas las áreas donde se desarrollan las actividades de sus habitantes.

Todos estos espacios deben ofrecer: bienestar, seguridad, conectividad, orden, accesibilidad universal, servicios básicos, proporción y escala, dadas estas condiciones que toda ciudad saludable debería tener en condiciones y parámetros alineados a un 100\%. No es así en la realidad a la que nos enfrentamos. por ello es importante contribuir desde el Observatorio Urbano Arquitectónico de Entornos Saludables a generar información y datos que ayuden a los organismos especializados, a la comunidad académica y a los gobiernos a tomar decisiones urbanas y arquitectónicas en los procesos de planeación, específicamente en el área de estudio planteada: la Zona Metropolitana Orizaba y Zona Metropolitana Córdoba, en las cuales se pueden identificar problemáticas en los aspectos antes descritos, se necesita una observación y medición específica de este fenómeno.

El observatorio pretende realizar mediciones y cálculo de indicadores que identifiquen con claridad los porcentajes de espacios saludables con los que contamos en las dos zonas metropolitanas. La observación, el registro y la medición del fenómeno ayudarán en las estrategias urbanas y arquitectónicas del diseño de espacios y entornos saludables; a nivel regional, nacional e internacional, contribuyendo como Universidad Veracruzana a presentar información confiable y sistematizada bajo el diseño de instrumentos de medición basados en metodologías de fuentes como INEGI.

Con el objetivo de fomentar la importancia de la cultura de la estadística en la aplicación y generación del conocimiento en los académicos y en los estudiantes, así como también concientizar a la comunidad Universitaria de la Responsabilidad Social Universitaria ante un problema común al cual la sociedad y las ciudades nos enfrentamos a diario ya que todas las actividades cotidianas las desarrollamos en los entornos urbanos y arquitectónicos.

El objeto de estudio del OUAES es identificar y monitorear los ambientes no saludables y los saludables (Espacios Arquitectónicos Urbanos). Teniendo un alcance territorial en dos zonas metropolitanas, ubicadas en la región 3 del estado de Veracruz. Estas son: la Zona Metropolitana Orizaba, que abarca 11 municipios; y la Zona Metropolitana Córdoba, la cual tiene 4 municipios. El fenómeno que se quiere abordar es la resiliencia en la percepción espacial urbana (percepción espacial urbana y arquitectónica de los espacios no saludables). 
El alcance territorial se determina con esta estructura según la definición global de la salud, Latapi (2015) menciona que: "Los municipios son los encargados de proveer todos los bienes y servicios que garantizan los fundamentos del bienestar de la población: servicios, urbanismo, vivienda, parques, recreación, cultura, vialidad, regulación, sistema tributario, servicios de salud y educación".

\section{¿Cuál es el objetivo principal del OUAES?}

El principal objetivo del Observatorio Urbano Arquitectónico de Entornos Saludables es contar con un espacio institucional, académico y confiable de insumos para el apoyo a la investigación. Se pretende recopilar, monitorear, analizar y generar información estadística relacionada con la observación del fenómeno que producen las ciudades no saludables relacionada al proceso de resiliencia urbana y arquitectónica, compartiendo conocimiento especializado para entender las ciudades como sistemas sociales y urbanos que inciden directamente en la salud y bienestar de los habitantes.

El Observatorio Urbano Arquitectónico de Entornos Saludables busca dar respuesta a las siguientes cuestiones:

1. ¿Qué tan saludable en la ciudad en donde habito?

2. ¿Es saludable la ZMO y ZMC?

3. ¿Por qué no es saludable?

4. ¿Cuáles son los indicadores que están generando los porcentajes más bajos para que la ZMO y ZMC no sean entornos saludables?

5. ¿Qué elementos y proyectos de Diseño Urbano y Arquitectónico se deberían realizar en la ZMO y ZMC para que fueran consideradas como zonas saludables?

La misión del Observatorio Urbano Arquitectónico de Entornos Saludables es tener un espacio académico de investigación que genera, analiza, compila y divulga información estadística pertinente y confiable referente a los indicadores que intervienen en los procesos de deterioro de las urbes generando entornos no saludables de la región tres del estado de Veracruz, comprendida por la Zona Metropolitana Córdoba y la Zona Metropolitana Orizaba. Aportando resultados a la comunidad universitaria, siendo socialmente responsable de los datos recopilados, monitoreados y generados para contribuir como una base de datos de calidad y confiables contribuyendo a la generación, aplicación y divulgación del conocimiento. El OUAE tiene el reto y la oportunidad de proyectar una visión multidireccional y multidisciplinar 


\section{Observatorios - Observatorio Urbano Arquitectónico de Entornos Saludables}

enfocado a ser un referente estatal, nacional e internacional que contribuya y aporte información de calidad y validez colegiada a los organismos relacionados con el fenómeno de estudio. El OUAES se visualiza al 2030 contribuyendo al logro de las metas de los ODS y generando información socialmente responsable para la Universidad Veracruzana como una Institución Pública de Educación Superior.

Los principios y valores del Observatorio Urbano Arquitectónico de Entornos Saludables, bajo los cuales desarrollará su trabajo, son de acuerdo con el código de ética de la Universidad Veracruzana: responsabilidad, respeto, honestidad, integralidad, transparencia, seguridad y cuidado, objetividad, libertad, equidad.

\section{Ejes estratégicos}

Los ejes Estratégicos del Observatorio se apegan a la pertenencia y pertinencia del Plan Estratégico 2017-2020, contribuyendo a las políticas institucionales planteadas. Considerando y visualizando las estrategias, metas y acciones del observatorio a la contribución directa de las metas institucionales con incidencia pertinente en los 3 ejes estratégicos. Así también, se alinea a la contribución de las metas de los ODS 2030 que se ligan directamente al fenómeno de estudio.

En relación con los ejes estratégicos institucionales en los que se contribuye con el OUAES son:

Liderazgo académico (Formación integral, uso de tecnologías de información y aprendizaje, fortalecimiento de cuerpos académicos, proyectos de investigación, consolidar estructuras colegiadas de investigación). Con el objetivo estratégico de contribuir con el trabajo de investigación a la formación integral, uso de tecnologías de información y aprendizaje, fortalecimiento de cuerpos académicos, proyectos de investigación y consolidación de estructuras colegiadas de investigación.

Visibilidad e impacto social (Sustentabilidad y promoción de la salud). Teniendo con el objetivo estratégico de contribuir a las estrategias y metas institucionales dirigidas a la sustentabilidad y a la promoción de la salud.

Gestión y Gobierno (Responsabilidad Social Universitaria RSU, desarrollo de proyectos de desarrollo institucional y académicos de investigación con recursos, innovación y desarrollo de la tecnología aplicada al aprendizaje y a la investigación, uso y diseño de infraestructura tecnológica). Con el objetivo estratégico de Contribuir en los programas de responsabilidad 
social universitaria, desarrollo de proyectos de desarrollo institucional y académicos de investigación con recursos, innovación y desarrollo de la tecnología aplicada al aprendizaje y a la investigación, uso y diseño de infraestructura tecnológica.

Contribución a los ODS 2030 de la ONU con el objetivo estratégico de contribuir de manera confiable y valida con la información generada en el Observatorio Urbano Arquitectónico de Entornos Saludables al logro de las metas de los ODS 2030; principalmente a los siguientes:

- $\quad$ Objetivo 3. Salud y Bienestar. Garantizar una vida sana y promover el bienestar de todas a todas las edades.

- Objetivo 4. Educación de calidad. Garantizar una educación inclusiva y equitativa de calidad y promover oportunidades de aprendizaje permanente para todos.

- Objetivo 11. Ciudades y comunidades sostenibles. Lograr que las ciudades y asentamientos humanos sean inclusivos, seguros, resilientes y sostenibles.

- Objetivo 13. Acción por el clima. Adoptar medidas urgentes para combatir el cambio climático y sus efectos.

\section{Glosario de términos básicos del OUAES}

En lo que refiere a los conceptos básicos del OUAES se identifican los siguientes, el cual es parte de un glosario de término que sirve como marco conceptual del fenómeno a observar.

Ciudad: Es un organismo complejo que vive, respira, crece y cambia de manera constante. OMS (1995)

Ciudad saludable: Es aquella que se crea de manera continua, que mejora sus ambientes físicos y sociales y amplía sus recursos para la comunidad, para que las personas puedan apoyarse mutuamente en la realización de todas las funciones de la vida y el desarrollo de su máximo potencial. Latapí (2014).

SMAES: Sociedad Mexicana de Arquitectos Especializados en Salud.

Municipio: Es el espacio territorial, social y político en donde se identifican y se manifiestan los problemas, inquietudes y necesidades de las comunidades que los habitan. Latapí (2014).

Municipio saludable: Es aquel donde las autoridades políticas, civiles, las instituciones, organizaciones públicas y privadas, empresarios, trabajadores y sociedad en general dedican constantes esfuerzos para mejorar las condiciones de vida, trabajo y cultura de la población, establecen una relación armoniosa con el ambiente físico y natural y expanden los recursos comunitarios para mejorar la convivencia, desarrollar la solidaridad, la cogestión y la democracia. Latapí (2014). 


\section{Observatorios - Observatorio Urbano Arquitectónico de Entornos Saludables}

Medio ambiente: Concepto relacionado a los factores ambientales físicos, biológicos, de contaminación atmosférica, de contaminación química, tanto del suelo, agua y aire y los factores socio-culturales y psicosociales relacionados con la vida en común. (Informe Lalonde 1974).

Entornos saludables: Son espacios territoriales de diferentes escalas que mantienen un equilibrio vital entre los factores que inciden en la vida cotidiana generando ambientes benéficos a los habitantes. Latapi (2015).

Entorno Urbano: Conjunto de elementos naturales construidos, que conforman el territorio urbano y que constituyen el marco de referencia y convivencia de los habitantes y visitantes determinado por las características físicas, costumbres y usos que se relacionan entre sí. Latapí (2014)

RSU: Responsabilidad socialmente universitaria.

\section{¿Qué tipo de investigación y datos genera el Observatorio Urbano Arquitectónico de entornos saludables?}

El OUAES es un observatorio académico de investigación y observación. El fenómeno que se quiere abordar es la resiliencia en la percepción espacial urbana y arquitectónica de los espacios no saludables, considerando lo que la OMS refiere a que la salud es un estado de perfecto bienestar físico, mental y social, y no sólo la ausencia de enfermedad. Por eso, para el OUAES es importante generar investigación académica y datos relacionados directamente al fenómeno de estudio. Los productos que se generarán desde este observatorio serán los siguientes:

1. Diseño de Instrumentos de obtención de datos básicos y derivados.

2. Cálculo de indicadores de entornos no saludables con los datos obtenidos de las encuestas diseñadas por el Observatorio Urbano Arquitectónico de Entornos Saludables; estadística de resultados y cartografía.

3. Catálogo de fichas de Proyectos de Regeneración Urbana ubicados en la Región 3 comprendida por la ZMC y ZMO (Georreferenciación en cartografía).

4. Catálogo de fichas de Edificios Históricos patrimonio ubicados en la Región 3 comprendida por la ZMC y ZMO. (Georreferenciación en cartografía).

5. Elaboración de Infografía con temática del Observatorio Urbano Arquitectónicos de Entornos Saludables para alimentar la página web oficial.

6. Generar un catálogo de recomendaciones urbanas y arquitectónicas a las problemáticas identificadas dependiendo de cada indicador analizado por zona metropolitana y por AGEB.

7. Indicadores y análisis de casos relacionados a las siguientes temáticas correlacionadas con los entornos saludables y la percepción espacial: Diseño y regeneración urbana, patrimonio 
histórico, vulnerabilidad y zonas de riesgo por factores intrínsecos y extrínsecos, vivienda, espacio público, bioclimatismo, planeación y diseño urbano, peritaje urbano, infraestructura, movilidad en entornos saludables, sustentabilidad.

\section{Sistemas de indicadores del OUAES}

Las constantes del fenómeno observado están definidas por los siguientes indicadores: Salud integral, percepción espacial, ambientes saludables y los 3 componentes que se consideran para identificarlos: la esperanza de vida, la percepción de bienestar y la huella ecológica. Las variables que inciden en el análisis y monitoreo del fenómeno son: el tiempo de permanencia en los espacios y las características urbanas contextuales.

Los indicadores que se consideran en materia de salud serán:

- Indicadores indirectos vinculados con la población rural y urbana; e indicadores directos vinculados con servicios y con infraestructura.

- Indicadores a nivel internacional serán directamente ligados los de la OMS, OPS, OCDE, Sustainable Development Report 2019, SDG Index and Dashboard Report 2017.

- Indicadores a nivel nacional que utiliza el OUAES: INEGI, SS, INSP e IMSS.

Los instrumentos que el OUAES utiliza análisis para la generación de la información estadística y está basada en estadística básica y derivada. En el caso de la aplicación de la estadística básica, refiere al diseño de encuestas específicas para el fenómeno y el levantamiento de información por muestreo. Considerando como unidad de muestreo el AGEB.

Y en el caso de la aplicación de la estadística derivada es el uso de los registros estadísticos y bancos de datos de fuentes confiables nacionales e internacionales. El OUAES basa sus instrumentos en la metodología de INEGI y Sustainable Development Report 2019, SDG Index and Dashboard Report 2017.

El observatorio utiliza fuentes de información para la correlación e interpretación de datos; considerando en la observación del fenómeno de estudio las siguientes encuestas: ENVIPE 2017, ENCIG 2017, MOPRADEF, MOLEC, MODUCULT y MOPRADEF; ya que se identifican datos relevantes asociados al fenómeno de observación y monitoreo. 


\section{Batería y clasificación de Indicadores del Observatorio Urbano Arquitectónico de Entornos Saludables OUAES}

El OUAES estructura su observación mediante la siguiente clasificación de indicadores. La batería de indicadores está organizada en tres tipos de indicadores.

- Indicadores básicos que determinan una ciudad saludable según Latapi, Jaime (2015) Ambiente físico de alta calidad, limpio y seguro; calidad en la vivienda; ecosistema estable y sostenible a largo plazo; una comunidad cohesionada y no explotable; alto grado de participación y control de los ciudadanos sobre las decisiones que afectan su vida; salud y bienestar; satisfacción de las necesidades básicas de alimento, agua, vivienda, ingresos, seguridad y trabajo para todos los habitantes; acceso a la variedad de experiencias y recursos que faciliten el contacto, interacción y comunicación de sus habitantes; economía diversa, vital e innovadora; fomento de la conexión con las culturas del pasado, la herencia biológica y con otros grupos e individuos; acceso para todos a los servicios de salud; elevado estado de salud y bajos niveles de enfermedad.

- Indicadores clave principales son los que nos darán la referencia o marco teórico para poder generar los cálculos estadísticos del OUAES. Los Indicadores claves refieren fórmula especifica e identificada por: INEGI, Happy Planet Index y Sustainable Development Report 2019, SDG Index and Dashboard Report 2017. Índice de rezago social, Índice de huella ecológica. Índice de esperanza de vida. Índice de percepción de bienestar. Índice de felicidad, (según Happy Planet Index se mide con tres componentes: la esperanza de vida, la percepción de bienestar y la huella ecológica), Indicadores indirectos vinculados con la población rural y urbana. Indicadores directos vinculados con servicios e infraestructura. Factores de riesgos a la salud en espacios abiertos y cerrados. Indicadores referidos en la carta de Ottawa (1986) indispensables para la salud: paz, techo, educación, comida, ingreso, ecosistema estable, recursos sostenibles, justicia social, equidad.

- Indicadores extensivos que se generarán por medio de encuestas y cuestionarios con obtención de datos estadísticos derivados y básicos. Servirán como una base de datos para poder generar los cálculos estadísticos de correlación de variables y constantes para obtención de porcentajes requeridos para la medición de los entornos saludables. El cálculo se realizará con la unidad mínima espacial definida como AGEB. Servicios básicos, Infraestructura, Estructura vial, Mobiliario urbano, Espacios públicos, Vegetación y áreas verdes, Accesibilidad Urbana, Percepción espacial, Legibilidad urbana, Sistemas de acceso, Territorio, Patrimonio Histórico, Vulnerabilidad espacial, Equipamiento urbano, Vivienda, Factores ambientales y entorno, Estilo de vida, Factores biológicos. 


\section{Metodología del OUAES}

El Observatorio Urbano Arquitectónico de Entornos saludables diseñó una metodología basada en la observación del fenómeno de forma cuantitativa y cualitativa la cual se basa en diseño de análisis con estadística básica y derivada para generar inferencias al fenómeno observado. La metodología del OUAES diseñó la siguiente estructura:

1. Contextualización del fenómeno de observación con una unidad de medida: AGEB.

2. Diagnostico (Mediante el reconocimiento del AGEB).

3. Aplicación de instrumento de obtención de datos: Si es a escala arquitectónica se aplica la encuesta denominada: "Encuesta semestral de Entornos Saludables Arquitectónicos ESESEES” y si la Escala es Urbana (Región 3 que comprenden la Zona Metropolitana Orizaba y Zona Metropolitana Córdoba) se aplica la encuesta denominada "Encuesta Semestral de Entornos Saludables Urbanos ESES-EURB".

4. Análisis, interpretación y correlación de los indicadores de la estadística básica y de la derivada.

5. Resultados de aplicación instrumento de muestreo.

6. Implementar un POE (Post Ocupancy Evaluation).

En lo que refiere a las escalas metodológicas del OUAES para la observación y monitoreo del fenómeno: Entornos no saludables. Define las siguientes:

1. Estrategias de recolección de datos: participación ciudadana, recorridos de exploración, encuestas, recorridos urbanos por AGEB con el uso de equipo especializado: Drone Mavic Pro.

2. Unidades de medición: Edificios, espacios determinados por poligonales definidas, AGEB, regiones y/o zonas metropolitanas.

3. Mapeo de exploración y diagnóstico.

4. Estrategia de análisis de datos y cálculo de indicadores.

5. Elaboración de matriz de resultados y cálculo de indicadores.

6. Elaboración de gráficas comparativas y analíticas.

7. Cartografía con representación temática por indicador.

8. Cartografía síntesis de correlación de indicadores e interpretación. 


\section{Observatorios - Observatorio Urbano Arquitectónico de Entornos Saludables}

El Diseño estadístico del OUAES tiene como objetivo generar estadística básica y derivada que permita conocer en corto plazo el comportamiento de un espacio no saludable, así como los principales indicadores que lo generan a escala urbana y/o escala arquitectónica. El observatorio estructura su diseño estadístico de la siguiente manera:

- Tamaño de muestra: Escala Urbana Región 3 del Estado de Veracruz. Comprende la Zona Metropolitana Orizaba, que se encuentra integrada por 11 municipios, y la Zona Metropolitana Córdoba, integrada por 4 municipios.

- Unidad de Observación: AGEBS

- Marco muestral: INEGI y Happy Planet Index y Sustainable Development Report 2019, SDG Index and Dashboard Report 2017. Estadística derivada.

- Cobertura / Periodo: Temporal semestral (Cada 6 meses).

- Grupo de población: Toda la población que habita en un AGEB.

- Lista de indicadores: Se pretende monitorear los siguientes indicadores relacionados con el fenómeno. Indicadores básicos, Indicadores clave principales e Indicadores extensivos. Salud integral, percepción espacial, ambientes saludables, esperanza de vida, percepción de bienestar, huella ecológica, servicios e infraestructura básica, accesibilidad urbana, percepción del espacio y legibilidad urbana, sociología urbana, espacios públicos, patrimonio histórico, vulnerabilidad espacial, áreas verdes y parques, habitabilidad.

\section{Conclusión}

El Observatorio Urbano Arquitectónico de Entornos Saludables contribuirá a la investigación, la docencia y a organismos regionales, nacionales e internacionales como un referente de observación urbano-arquitectónico relacionado a los entornos no saludables. En donde nuestra aportación de contribuir en generar información, y monitorear el fenómeno, contribuya de manera pertinente a la región, a la Universidad Veracruzana y a nuestro país. En donde nuestra aportación en generar bancos y agenda de datos sirvan para otros organismos, académicos y estudiantes en la toma de decisiones, en las planificaciones estratégicas urbanas, en los planes de desarrollo urbano y regional; así como en investigaciones de tesis sobre casos de estudio relacionados a las temáticas de observatorio y a las problemáticas identificadas en el territorio de observación. Es un reto y una visión del OUAES el contribuir a la institución y fortalecer e impulsar la investigación con la generación de evidencias de calidad, así como su divulgación. Considerando y manteniendo alineado el observatorio al Plan estratégico 2017-2020 de la Universidad Veracruzana, contribuyendo con las estrategias, metas y acciones con resultados que aporten, a su vez, una contribución a las metas de los ODS 2030. 
Es importante para el OUAES, a nivel nacional e internacional, poder aportar y contribuir con acciones de investigación académica que generen conocimiento para colaborar en indicadores de salud asociados con la arquitectura y el urbanismo, entornos y ciudades saludables, ciudades y comunidades sostenibles, educación de calidad.

\section{Referencias}

Latapí, J. (2015). Ciudades Saludables en el contexto internacional. Primera edición.

Recuperado de

https://www.anmm.org.mx/publicaciones/CAnivANM150/L26_ANM_Ciudades_saludabl es.pdf

Latapí, J. (2014). Hacia un entorno Saludable en las comunidades mexicanas. Conacyt. Recuperado de https://www.anmm.org.mx/publicaciones/CAnivANM150/L17-Hacia-unentorno-saludable-en-las-cominidades-mexicanas.pdf

Lalonde, M. (1981). A new perspective on the health of canadadians. Informe Lalonde. Recuperado de https://Www.gob.mx/cms/uploads/attachment/file/10204/informelalonde.pdf

OMS (1995). Veinte pasos para desarrollar un proyecto de ciudades saludables. Recuperado de https://es.slideshare.net/Alejxandra/pasos-proyecto-ciudad-saludable

WHO (1998) Promoción de la Salud. Glosario. Recuperado de https://apps.who.int/iris/bitstream/handle/10665/67246/WHO_HPR_HEP_98.1_spa.pdf?s equence $=1$ 